Research Article

\title{
Cortex Cercis chinensis Granules Attenuate Streptococcus pneumoniae Virulence by Targeting Pneumolysin
}

\author{
Yan Xu, ${ }^{1}$ Yanbo Wang, ${ }^{1}$ Yinan Guo, ${ }^{2}$ Lina Wei, ${ }^{2}$ Lizhong Ding, ${ }^{2}$ Zhongtian Wang $\mathbb{D},{ }^{1}$ \\ and Liping Sun $\mathbb{D i}^{1}$ \\ ${ }^{1}$ Changchun University of Chinese Medicine, Changchun, Jilin 130117, China \\ ${ }^{2}$ Affiliated Hospital of Changchun University of Chinese Medicine, Changchun, Jilin 130021, China \\ Correspondence should be addressed to Liping Sun; slpcczyydx@sina.com
}

Received 12 February 2020; Revised 3 May 2020; Accepted 12 May 2020; Published 16 June 2020

Guest Editor: Lei Xu

Copyright ( 92020 Yan Xu et al. This is an open access article distributed under the Creative Commons Attribution License, which permits unrestricted use, distribution, and reproduction in any medium, provided the original work is properly cited.

Pore-forming toxins produced by bacteria are some of the most important molecular weapons for bacterial virulence. Pneumolysin (PLY) is a pore-forming toxin secreted by Streptococcus pneumoniae (S. pneumoniae) and plays a vital role in the spread, colonization, and invasion of this bacterium in the host, indicating that PLY is a promising target for developing treatments against S. pneumoniae infection. In this study, Cortex Cercis chinensis granules (CCCGs), a prescription drug on the market, were shown to inhibit the pore-forming activity of PLY and protect against PLY-mediated cell hemolysis and A549 cell death without antibacterial activity or inhibition of PLY production. In addition, CCCG treatment inhibited the oligomerization of PLY. Animal experiments showed that CCCGs can reduce the death of mice infected with S. pneumoniae, the degree of pathological damage to the lungs, and the levels of TNF- $\alpha$ and IL- 6 in the lungs. In summary, our results demonstrated that CCCGs, a marketed Chinese medicine, inhibit PLY activity and subsequently attenuate $S$. pneumoniae virulence, which would offer a novel strategy for fighting S. pneumoniae infection and a new use for CCCGs.

\section{Introduction}

Pore-forming toxins (PFTs) produced by bacteria act on the plasma membrane of eukaryotic cells and form a pore structure on the cell membrane, thus disrupting the concentration of liquid inside and outside the cell and causing cell swelling and cell lysis [1]. Pneumolysin (PLY) is such a toxin secreted by Streptococcus pneumoniae (S. pneumoniae) and is a multifunctional protein consisting of 471 amino acids with a molecular weight of $53 \mathrm{kDa}$. During the establishment of $S$. pneumoniae infection, PLY in the cytoplasm cannot be directly secreted into the extracellular space. However, after cell wall lysis by autolysin, antibiotic action, or the host-mediated immune response, PLY can be released extracellularly to exert its pore-forming activity [2]. Furthermore, 34-50 PLY monomer molecules can form oligomerized anterior pore complexes and bind to the membrane cholesterol to form a barrel-shaped transmembrane pore of approximately $25 \mathrm{~nm}$ in diameter, thereby rupturing the cell [3]. In addition, the release of PLY can also facilitate S. pneumoniae evasion of host defenses [4] and trigger acute lung injury and pulmonary fibrosis through direct cytotoxicity and indirect proinflammatory effects $[5,6]$. Furthermore, the production of PLY increases the incidence of acute cardiac events [7] and the risk of otitis media [8], meningitis [9], and even death [2]. Given the key role of PLY in the pathogenesis of $S$. pneumoniae-related organ damage and dysfunction caused by invasive pneumococcal disease (IPD), loss of PLY can significantly reduce the number of $S$. pneumoniae adhering to the human body and reduce IPD [10]. Thus, targeting PLY may be a promising anti-infection strategy to treat $S$. pneumoniae infection or improve the efficacy of antibiotics.

Agents directed against this toxin include murine monoclonal antibodies, PLY-4 and PLY-7, which target various epitopes on the toxin, blocking the binding to eukaryotic cell membranes and cytolytic activity [11]. Additionally, $\beta$-sitosterol [12], apigenin [13], and several other 
plant-derived agents $[14,15]$ have been reported to inhibit the oligomerization of PLY monomers. The problem is that none of these antibodies or inhibitors can be used in clinical or even preclinical research. Therefore, we tried to find a new drug that inhibits PLY activity from among Chinese medicine granules, which have been used for thousands of years with effectiveness and safety in bodily experiment verification for long-term use in humans. Our research is an attempt to explore new therapeutic effects or clinical use of traditional Chinese medicine particles from an experimental perspective.

Cortex Cercis chinensis (CCC), a Chinese medicine called ZI JINGPI, is the dry root bark of the Magnoliaceae plant Kadsura longipedunculata Finet et Gagnep. CCC is derived from "the Materia Medica of Rihuazi," a medical book of the five dynasties of China, which is reported to have the effects of promoting blood circulation and meridian circulation, reducing swelling, and detoxifying and is used for the treatment of irregular menstruation, such as amenorrhea, dysmenorrhea, and bloating, worms, and snake bites. Here, we further found that Cortex Cercis chinensis granules (CCCGs), a commercial product of CCC, possess inhibitory effects against PLY activity and S. pneumoniae virulence.

\section{Materials and Methods}

2.1. Cells and Bacteria. Alveolar epidermal cells (A549) were obtained from the ATCC (Manassas, VA) and cultured in DMEM with $10 \%$ fetal bovine serum (FBS, Biological Industries, Israel).

Streptococcus pneumoniae strain D39 serotype 2 (NCTC 7466) was kindly provided by Professor Jian Huang from Zunyi Medical University (Zunyi, China) and cultured in the Todd Hewitt broth with $1 \%$ yeast extract (THY media) at $37^{\circ} \mathrm{C}$ with $5 \% \mathrm{CO}_{2}$.

2.2. Cortex Cercis chinensis Granules. CCCGs were purchased from Jiangyin Tianjiang Pharmaceutical Co., Ltd., (Jiangyin, China), and the production batch number was 19046614. CCCGs are a traditional Chinese medicine granule prescription drug approved by the China Food and Drug Administration that have been verified to meet the required standard of the China Food and Drug Administration. This drug was dissolved in DMSO for the following use.

2.3. Antibacterial Activity Determination. The minimum inhibitory concentration of CCCGs against the S. pneumoniae strain D39 was examined according to a previous study [16]. The growth of the S. pneumoniae strain D39 treated with various concentrations $(0,4,8,16$, or $32 \mu \mathrm{g} / \mathrm{ml})$ of CCCGs was determined by monitoring the OD600 $\mathrm{nm}$ of each sample every $30 \mathrm{~min}$.

2.4. Recombinant Pneumolysin Preparation. Construction of a pET28a vector containing the ply sequence and the PLY protein purification methods were based on previous descriptions [17]. In short, harvested cells were lysed by sonication, and the supernatant of the centrifuged cell lysate was loaded onto a Ni-NTA agarose column. The target protein was flushed with an elution buffer (PBS containing $200 \mathrm{mM}$ imidazole, $\mathrm{pH}$ 7.4). Then, the recombinant protein was concentrated using a Millipore Amicon filter $(30 \mathrm{kDa}$ cutoff) for desalting.

2.5. Hemolysis Assay. Here, $1.0 \mu \mathrm{l}$ of purified PLY (0.2 mg/ $\mathrm{ml})$ in $965 \mu \mathrm{l}$ of PBS was treated with various concentrations $(0,4,8,16$, or $32 \mu \mathrm{g} / \mathrm{ml})$ of CCCGs at $37^{\circ} \mathrm{C}$ for $30 \mathrm{~min}$. Then, $25 \mu \mathrm{l}$ of defibrinated sheep red blood cells were added to the system and incubated at $37^{\circ} \mathrm{C}$ for $10 \mathrm{~min}$. Finally, after centrifugation at $3000 \times \mathrm{g}$ for $5 \mathrm{~min}, 200 \mu \mathrm{l}$ of the supernatant was pipetted to determine the hemolytic activity at $\mathrm{OD}_{543 \mathrm{~nm}}$. The sample treated with DMSO was used as $0 \mu \mathrm{g} / \mathrm{ml}$ CCCGs.

2.6. Immunoblot Analysis. S. pneumoniae D39 cells were grown with different concentrations of CCCGs $(0,4,8,16$, and/or $32 \mu \mathrm{g} / \mathrm{ml}$ ). An equal amount of bacterial culture supernatant from each sample was suspended in the same volume of the Laemmli sample buffer, boiled for $10 \mathrm{~min}$, and separated by $12 \%$ SDS-PAGE. Following transfer onto a PVDF membrane, the PLY protein was detected using a monoclonal antibody against PLY (1:1000, Abcam, Cambridge, UK) and HRP-conjugated secondary anti-mouse antibodies (1:2000, Proteintech) and developed with the ECL reagent (Thermo Scientific, Rockford, IL, USA).

2.7. Pneumolysin Oligomerization Analysis. Different concentrations of CCCGs $(0,4,8,16$, and/or $32 \mu \mathrm{g} / \mathrm{ml})$ were mixed with PLY at $37^{\circ} \mathrm{C}$ for $1 \mathrm{~h}$. Then, $5 \mathrm{x}$ SDS-PAGE loading buffer without $\beta$-mercaptoethanol was added and incubated for $10 \mathrm{~min}$ at $50^{\circ} \mathrm{C}$. Twenty microliters of the samples were separated by $6 \%$ SDS-PAGE and transferred onto a PVDF membrane. Then, the monomers or oligomers were detected using immunoblot analysis as described above. The sample treated with DMSO was used as $0 \mu \mathrm{g} / \mathrm{ml}$ CCCGs. The grey scales of the bands for PLY oligomers/monomers were analysed using Image-Pro Plus software.

2.8. Green/Red and Cytotoxicity Test. A549 cells were trypsinized and seeded at a density of $2 \times 10^{4}$ cells per well in 96well plates for the overnight culture. The cells were incubated for $5 \mathrm{~h}$ with PLY that was treated with or without CCCGs in a $37^{\circ} \mathrm{C}$ incubator. Cell viability was observed using live/dead (green/red) reagents (Invitrogen, Carlsbad, CA, USA) according to the manufacturer's instructions. Finally, images were acquired using a confocal laser scanning microscope (Olympus, Tokyo, Japan). A cytotoxicity detection kit (Roche, Mannheim, Germany) was used for the determination of lactate dehydrogenase (LDH) activity. Following centrifugation in a 96-well plate $(1000 \mathrm{rpm}, 10 \mathrm{~min})$, the supernatant was mixed with the reaction reagents for incubation for $30 \mathrm{~min}$ in the dark. Then, LDH activity was measured at $\mathrm{OD}_{492 \mathrm{~nm}}$ by a microplate reader (Tecan, 
Salzburg, Austria). The sample treated with DMSO was used as $0 \mu \mathrm{g} / \mathrm{ml} \mathrm{CCCGs.}$

2.9. Mouse Infection Assays. $\mathrm{BALB} / \mathrm{c}$ mice (female, 6-8 weeks old, 20-22 g) were purchased from Liaoning Changsheng Biotechnology Co., Ltd., (Shenyang, China) and maintained in accordance with the NIH Guide for the Care and Use of Laboratory Animals. All mouse experiments were approved by the Ethics Committee of the Changchun University of Chinese Medicine. The $S$. pneumoniae strain D39 was cultured in THB at $37^{\circ} \mathrm{C}$ until the $\mathrm{OD}_{600 \mathrm{~nm}}$ reached 0.4 (midlogarithmic phase), collected by centrifugation (1000 rpm for $10 \mathrm{~min}$ ), and washed three times with PBS. Except for those in the healthy control group, each mouse was nasally infected with $1.5 \times 10^{8}$ colony-forming units (CFUs) of bacteria. These mice were subcutaneously injected with CCCGs $(40 \mathrm{mg} / \mathrm{kg}$ ) or DMSO every $8 \mathrm{~h}$, and the mice were observed for survival for $72 \mathrm{~h}$ $(n=12)$. For other mouse infection assays $(n=8)$, the lungs from sacrificed mice were used for gross pathological analysis by a camera or histopathological analysis by hematoxylin-eosin staining under light microscopy at $48 \mathrm{~h}$ after infection. Bronchoalveolar lavage fluid was collected in two bronchial sterile PBS infusions, totaling $500 \mu \mathrm{l}$. After liquid centrifugation, TNF- $\alpha$ and IL-6 levels were measured according to the instructions of an ELISA kit (eBioscience, San Diego, CA, USA).

2.10. Statistical Analysis. The data were analyzed by GraphPad Prism 6.0 (GraphPad Software) using Student's ttest; ${ }^{*} p<0.05$ and ${ }^{* *} p<0.01$, and the data are expressed as mean $\pm \mathrm{SD}(n \geq 3)$.

\section{Results}

3.1. CCCGs Inhibit PLY-Mediated Hemolysis. We first performed a hemolysis test to screen for potential PLY inhibitors and found that CCCG treatment reduced PLY-mediated red blood cell hemolysis in a concentration-dependent manner, as shown in Figure 1(a). These results proved that CCCGs block the activity of PLY.

3.2. CCCGs Do Not Inhibit the Growth of S. pneumoniae or the Production of PLY. Next, we mapped the antibacterial profile of CCCGs. As shown in Figure 1(b), CCCGs did not directly inhibit the growth of $S$. pneumoniae at concentrations of $4,8,16$, or $32 \mu \mathrm{g} / \mathrm{ml}$. In addition, the minimum inhibitory concentration (MIC) of CCCGs against $S$. pneumoniae was no less than $512 \mu \mathrm{g} / \mathrm{ml}$. Then, we continued to verify whether CCCGs would affect PLY production in $S$. pneumoniae, and as shown in Figure 1(c), CCCGs had no effect on PLY production at every concentration (up to $32 \mu \mathrm{g} / \mathrm{ml}$ ) tested in this study. In summary, our results demonstrated that CCCGs can prevent PLY-mediated cell hemolysis without affecting the growth of $S$. pneumoniae or PLY production.
3.3. CCCGs Inhibit PLY Oligomerization. The above results suggested that CCCG treatment could directly inhibit the activity of PLY; however, the potential mechanism of such inhibition was not clear. Pore-forming activity is the main physiological function of PLY, which is closely associated with the oligomerization of PLY. Thus, we speculated that CCCGs achieve an inhibitory function by blocking the oligomerization of PLY. Western blot experiments confirmed our conjecture. As shown in Figure 2, CCCGs inhibited the oligomerization of PLY, thereby reducing the activity of PLY.

3.4. CCCGs Inhibit PLY-Mediated Death of A549 Cells. To determine whether CCCGs can inhibit the cytotoxicity of PLY at the cellular level, A549 human alveolar basal epithelial cells were selected for the following assays. After incubation with PLY that was treated with or without CCCGs, the cytotoxicity of A549 cells was observed. As shown in Figure 3(a), PLY incubation led to robust cell death, as evidenced by the fact that most cells were stained red. However, such red cells were significantly reduced following CCCG treatment (Figures 3(b) and 3(c)). Additionally, CCCG treatment exhibited no cytotoxicity against A549 cells (Figure 3(d)). In agreement with this observation, the cell viability was significantly increased for PLY-treated A549 cells in the presence of increasing concentrations of CCCGs (Figure 3(e)). Taken together, our results suggested that CCCG treatment provides robust protection against PLY-mediated cytotoxicity in A549 cells.

3.5. CCCGs Protect Mice from S. pneumoniae Infection. The in vitro inhibition of PLY activity by CCCGs prompted us to examine whether protection occurs in vivo. BALB/c mice were selected for animal experiment verification. After nasal infection of mice with $S$. pneumoniae, we found that CCCG treatment could protect mice from S. pneumoniaemediated death (Figure 4(a)), as evidenced by $16.67 \%$ survival and $58.33 \%$ survival for infected mice that received DMSO and CCCG, respectively. Moreover, compared with that in the lungs of the S. pneumoniae D39 infection group, the pathological damage in the lungs of the CCCG treatment group was significantly alleviated, with more ruddy and shiny appearance (Figure 4(b)). The pathological lung sections showed that the lung inflammation of mice was significantly reduced after CCCG treatment (Figure 4(b)), which is consistent with the results in Figure 4(b). Finally, we measured the levels of TNF- $\alpha$ and IL- 6 in bronchoalveolar lavage fluid in infected mice. As shown in Figure 4(d), CCCG treatment also reduced the amount of proinflammatory factors in the lungs of $S$. pneumoniae-infected mice. Taken together, our results established that CCCGs protected against $S$. pneumoniae infection in mice by inhibiting the pore-forming activity of PLY.

\section{Discussion}

PFTs are complex and widespread virulence factors produced by pathogenic bacteria that can destroy the host's 


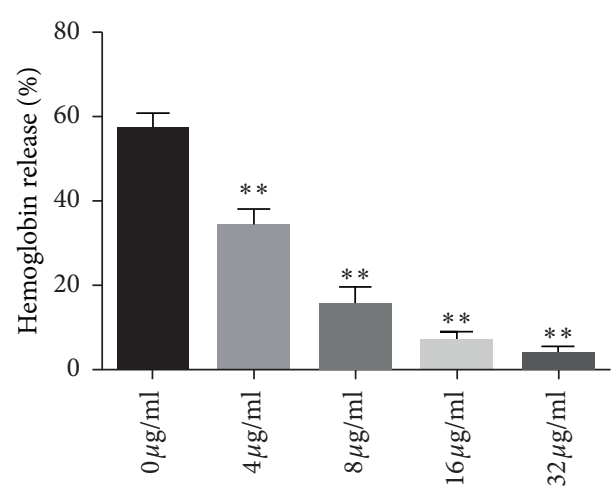

(a)

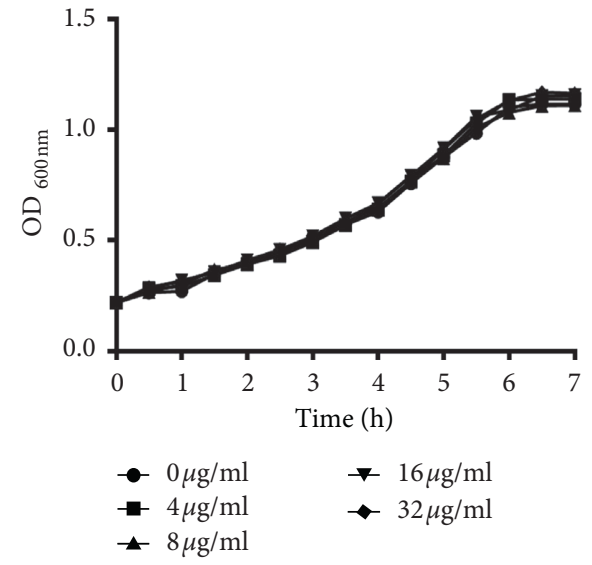

(b)

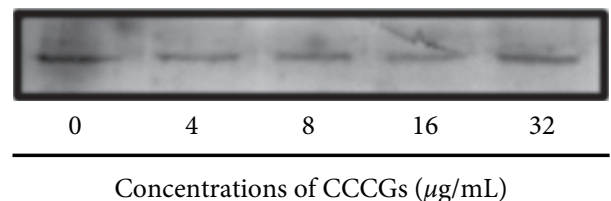

(c)

FIgURE 1: CCCGs inhibit PLY activity without affecting $S$. pneumoniae viability or PLY production. (a) PLY was pretreated with the indicated concentrations of CCCGs, and the activity of PLY was tested using a hemolysis assay. Data are presented as the mean \pm SD $(n=3)$. ${ }^{* *} p<0.01$. (b) S. pneumoniae was cocultured with various concentrations of CCCGs, and then the OD600 nm of each sample was determined every $30 \mathrm{~min}$. (c) S. pneumoniae was cocultured with various concentrations of CCCGs, and the PLY production in the bacterial culture supernatants was examined by immunoblot analysis.

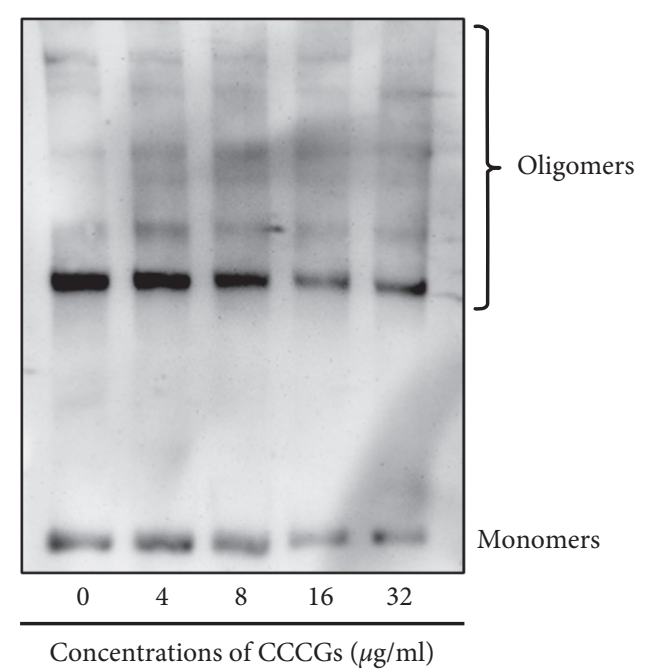

(a)

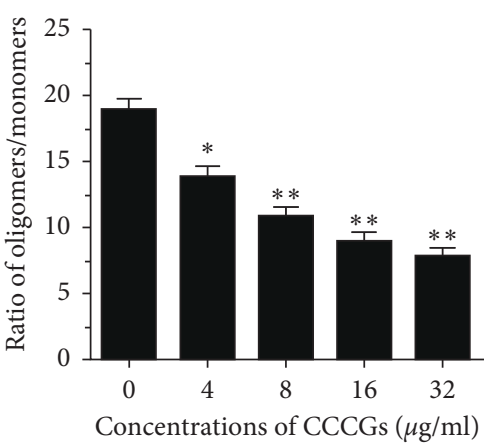

(b)

FIgURE 2: CCCGs reduce the oligomerization of PLY. (a) PLY was pretreated with the indicated concentrations of CCCGs, and monomers and oligomers were determined using immunoblot analysis. (b) The grey scales of the bands for PLY oligomers/monomers were analysed using Image-Pro Plus software. ${ }^{*} p<0.05 ;{ }^{* *} p<0.01$.

epithelial barrier, kill immune cells, and facilitate bacterial spread and multiplication [18]. Similar to most PFTs, PLY directly causes cell lysis at high concentrations and induces cell inflammation and apoptosis at low concentrations [19]. After the cell membrane interacts with a low concentration of PLY, cells can even self-repair the membrane by, for example, lysosomal decomposition after endocytosis [20] or directly discarding the pores from the cell [21], ultimately avoiding death. However, regardless of whether PLY can cause cell death, this toxin can greatly affect cell function, 


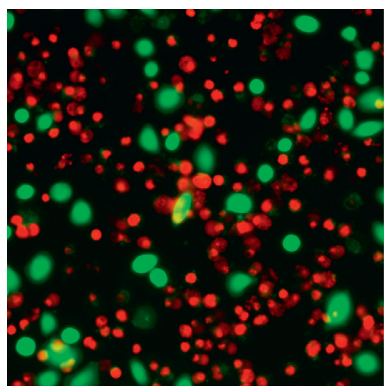

(a)

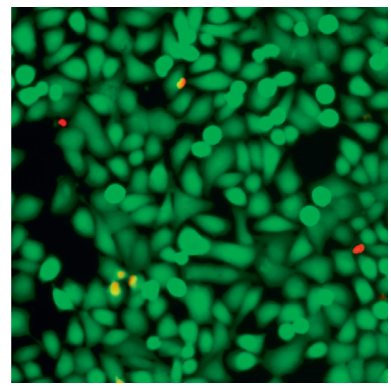

(d)

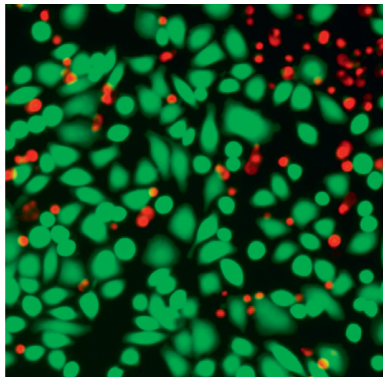

(b)

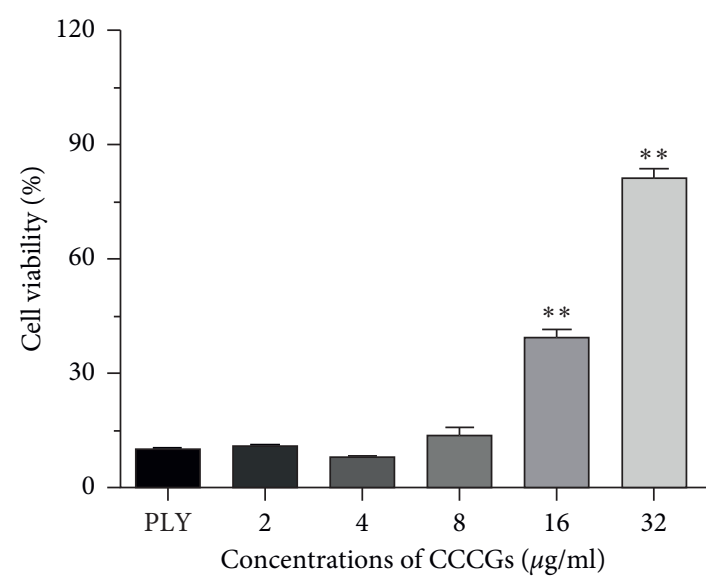

(e)

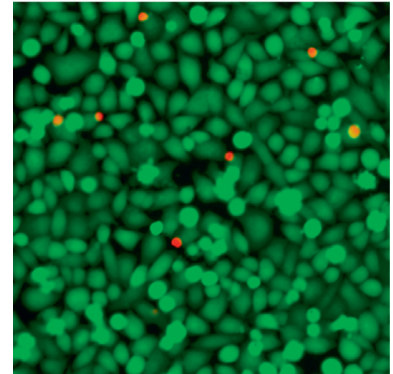

(c)

FIgURE 3: CCCGs attenuate PLY-mediated cytotoxicity. A549 cells were incubated for $5 \mathrm{~h}$ with PLY that was treated with or without CCCGs. Then, the cells were stained with live (green) or dead (red) reagent and observed under a confocal laser scanning microscope. (a) A549 cells cocultured with PLY; (b) A549 cells cocultured with PLY in the presence of $4 \mu \mathrm{g} / \mathrm{ml} \mathrm{CCCGs;} \mathrm{(c)} \mathrm{A549} \mathrm{cells} \mathrm{cocultured} \mathrm{with} \mathrm{PLY} \mathrm{in} \mathrm{the}$ presence of $32 \mu \mathrm{g} / \mathrm{ml} \mathrm{CCCGs;} \mathrm{and} \mathrm{(d)} \mathrm{A549} \mathrm{cells} \mathrm{cocultured} \mathrm{with} 32 \mu \mathrm{g} / \mathrm{ml} \mathrm{CCCGs.} \mathrm{(e)} \mathrm{A549} \mathrm{cells} \mathrm{were} \mathrm{incubated} \mathrm{for} 5 \mathrm{~h}$ with PLY that was treated with or without CCCGs, and the LDH released into the supernatants of the coculture system was determined using a cytotoxicity detection kit. Data are presented as mean $\pm \mathrm{SD}(n=3) .{ }^{* *} p<0.01$.

which is important for S. pneumoniae virulence. However, inhibition of PLY production may prompt S. pneumoniae to rapidly mutate to find opportunities for survival in the complex host internal environment. This "human-induced evolution" approach will instead stimulate S. pneumoniae to produce other invasins and virulence mechanisms, ultimately leading to human failure in this conflict [22]. Thus, direct neutralization of PLY activity instead of preventing its production or directly killing the bacteria becomes an ideal choice.

CCCGs have been reported to possess anti-inflammatory and bactericidal effects in ancient Chinese medical books. Here, we aimed to find a unique antibacterial drug from the treasure trove of the civilization of the Chinese nation, as Tu did [23]. On the other hand, CCCGs are a drug developed by good manufacturing practice (GMP) and have been used in hospitals and communities in China for many years. They have strict production processes from raw materials, decoction pieces, creams, intermediates, and finished products, with the production batch number listed in the materials and methods.

In this study, CCCGs were confirmed to have no direct antibacterial effect against $S$. pneumoniae at concentrations up to $32 \mu \mathrm{g} / \mathrm{ml}$. However, CCCG treatment significantly reduced PLY-mediated cytotoxicity through hemolysis experiments and live/dead cell experiments. Western blot analysis further demonstrated that CCCGs can neutralize PLY pore-forming activity without affecting PLY production by $S$. pneumoniae. Animal experiments further verified that CCCGs can protect mice infected with $S$. pneumoniae from death and significantly reduce lung inflammation and the release of proinflammatory factors. In short, all these experiments demonstrated that CCCGs can reduce the pathogenicity of $S$. pneumoniae by neutralizing the cytotoxicity of PLY. This anti-infection strategy by CCCGs does not put evolutionary pressure on $S$. pneumoniae.

Human technology and antibacterial strategies have continued to evolve, but this long-term enemy has not been completely eliminated. Antibacterial infection strategies should maintain a balance between bacteria and hosts but not employ a simple bactericidal action. As reported in our study, CCCGs are such a drug to fight bacterial infection without putting selective pressure on the targeted bacteria. The use of plants and their extracts to treat diseases is not a unique method only in China. Similar methods are available in India, South Korea, Japan, most African regions, and the 


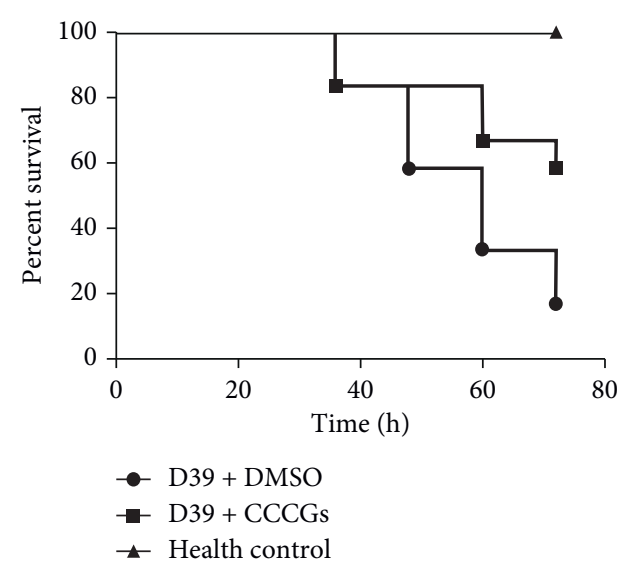

(a)

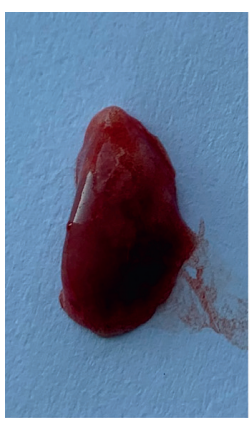

D39+DMSO

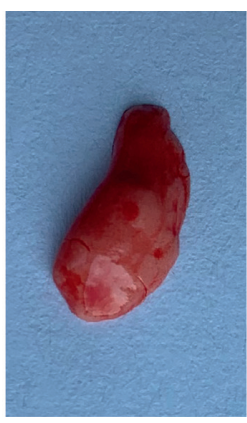

D39+CCCGs

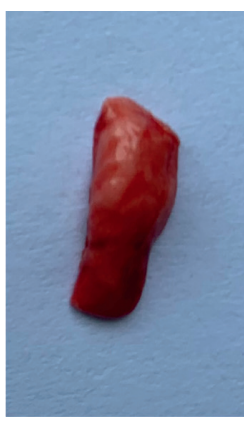

Control

(b)

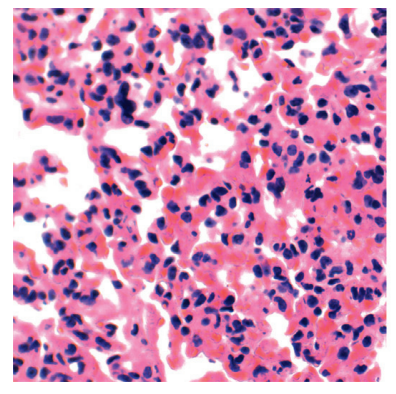

D39+DMSO

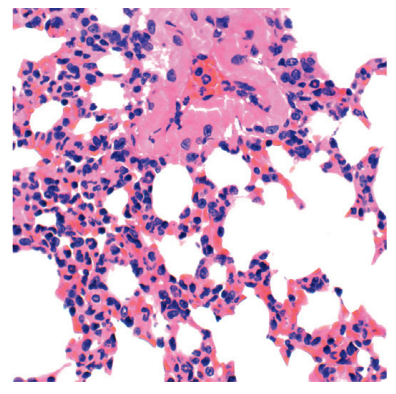

D39+CCCGs

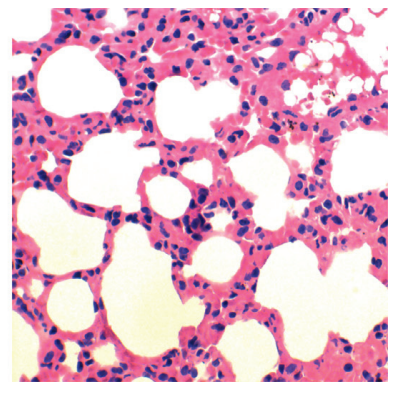

Control

(c)
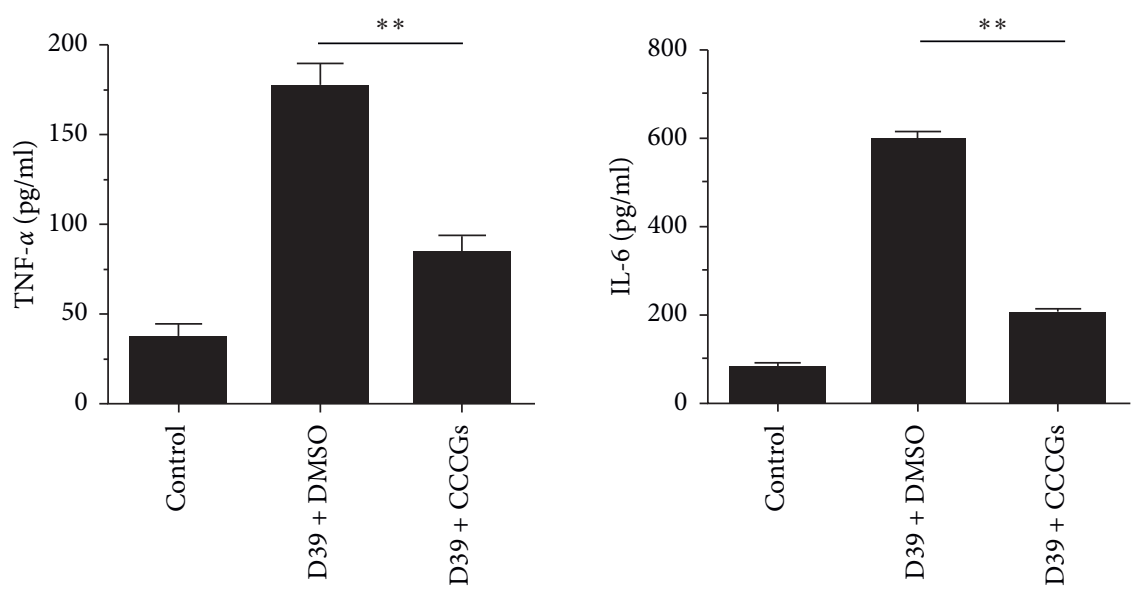

(d)

Figure 4: CCCGs protect mice from S. pneumoniae infection. BALB/c mice were nasally infected with $1.5 \times 10^{8} \mathrm{CFUs}$ of $S$. pneumoniae and treated with $40 \mathrm{mg} / \mathrm{kg}$ CCCGs or DMSO for $72 \mathrm{~h}$. (a) The survival of infected mice with the indicated treatment was monitored for $72 \mathrm{~h}$. The lungs from sacrificed mice were used for gross pathological analysis by (b) a camera or (c) histopathological analysis by hematoxylin-eosin staining under light microscopy at $48 \mathrm{~h}$ after infection. (d) The inflammatory factors (TNF- $\alpha$ and IL-6) in the bronchoalveolar lavage fluid were examined by ELISA. Data are presented as mean $\pm \mathrm{SD}(n=3) .{ }^{* *} p<0.01$.

Amazon. Research on plant-based antibacterial drugs may be the key research direction of supplementing and replacing antibiotics in the future.

In short, our study successfully used CCCGs as a blocker of PLY pore-forming activity and subsequently reduced the pathogenicity of $S$. pneumoniae, which demonstrated the potential of Chinese medicine to treat bacterial infection.

\section{Data Availability}

The experimental data used to support the findings of this study are included within the article.

\section{Conflicts of Interest}

The authors declare that they have no conflicts of interest. 


\section{Authors' Contributions}

Yan $\mathrm{Xu}$ and Yanbo Wang contributed equally to this work.

\section{Acknowledgments}

This work was supported by the National Key Research and Development Program of China (2017YFC1703202), the Jilin Scientific and Technological of Chinese Medicine Program (2019023), the Inheritance and Innovation of Chinese Medicine of "Millions of Standouts" Project (the Project of Qihuang), the Inheritance Workroom of the Chinese Medicine Master Wang Lie, and the Jilin Scientific and Technological Program of Sanitation and Population Control (2018J106).

\section{References}

[1] F. C. O. Los, T. M. Randis, R. V. Aroian, and A. J. Ratner, "Role of pore-forming toxins in bacterial infectious diseases," Microbiology and Molecular Biology Reviews, vol. 77, no. 2, pp. 173-207, 2013.

[2] T. J. Mitchell and C. E. Dalziel, "The biology of pneumolysin," MACPF/CDC Proteins-Agents of Defence, Attack and Invasion, vol. 80, pp. 145-160, 2014.

[3] K. van Pee, E. Mulvihill, D. J. Yildiz, and Ö. Yildiz, "Unraveling the pore-forming steps of pneumolysin from Streptococcus pneumoniae," Nano Letters, vol. 16, no. 12, pp. 7915-7924, 2016.

[4] Z. Zhang, T. B. Clarke, and J. N. Weiser, "Cellular effectors mediating Th17-dependent clearance of pneumococcal colonization in mice," The Journal of Clinical Investigation, vol. 119, no. 7, pp. 1899-1909, 2009.

[5] M. García-Suárez Mdel, N. Flórez, A. Astudillo et al., "The role of pneumolysin in mediating lung damage in a lethal pneumococcal pneumonia murine model," Respiratory Research, vol. 8, no. 1, p. 3, 2007.

[6] S. Knippenberg, B. Ueberberg, R. Maus et al., "Streptococcus pneumoniae triggers progression of pulmonary fibrosis through pneumolysin," Thorax, vol. 70, no. 7, pp. 636-646, 2015.

[7] D. M. Musher, A. M. Rueda, A. S. Kaka, and S. M. Mapara, "The association between pneumococcal pneumonia and acute cardiac events," Clinical Infectious Diseases, vol. 45, no. 2, pp. 158-165, 2007.

[8] M. Perny, M. Solyga, D. Grandgirard, M. Roccio, S. L. Leib, and P. Senn, "Streptococcus pneumoniae-induced ototoxicity in organ of Corti explant cultures," Hearing Research, vol. 350, pp. 100-109, 2017.

[9] D. W. Kim, P. E. Kilgore, E. J. Kim et al., "The enhanced pneumococcal LAMP assay: a clinical tool for the diagnosis of meningitis due to Streptococcus pneumoniae," PLoS One, vol. 7, no. 8, Article ID e42954, 2012.

[10] H. Marriott, T. Mitchell, and D. Dockrell, "Pneumolysin: a double-edged sword during the host-pathogen interaction," Current Molecular Medicine, vol. 8, no. 6, pp. 497-509, 2008.

[11] M. García-Suárez Mdel, M. D. Cima-Cabal, N. Flórez et al., "Protection against pneumococcal pneumonia in mice by monoclonal antibodies to pneumolysin," Infection and Immunity, vol. 72, no. 8, pp. 4534-4540, 2004.

[12] H. Li, X. Zhao, J. Wang et al., “ $\beta$-sitosterol interacts with pneumolysin to prevent Streptococcus pneumoniae infection," Scientific Reports, vol. 5, no. 1, p. 17668, 2015.
[13] M. Song, L. Li, M. Li, Y. Cha, X. Deng, and J. Wang, “Apigenin protects mice from pneumococcal pneumonia by inhibiting the cytolytic activity of pneumolysin," Fitoterapia, vol. 115, pp. 31-36, 2016.

[14] X. Zhao, H. Li, J. Wang et al., "Verbascoside alleviates pneumococcal pneumonia by reducing pneumolysin oligomers," Molecular Pharmacology, vol. 89, no. 3, pp. 376-387, 2016.

[15] X. Zhao, B. Liu, S. Liu, L. Wang, and J. Wang, "Anticytotoxin effects of amentoflavone to pneumolysin," Biological \& Pharmaceutical Bulletin, vol. 40, no. 1, pp. 61-67, 2017.

[16] J. Qiu, X. Niu, J. Dong et al., "Baicalin protects mice from Staphylococcus aureus pneumonia via inhibition of the cytolytic activity of $\alpha$-hemolysin," The Journal of Infectious Diseases, vol. 206, no. 2, pp. 292-301, 2012.

[17] M. Song, Z. Teng, M. Li, X. Niu, J. Wang, and X. Deng, "Epigallocatechin gallate inhibits Streptococcus pneumoniae virulence by simultaneously targeting pneumolysin and sortase A," Journal of Cellular and Molecular Medicine, vol. 21, no. 10, pp. 2586-2598, 2017.

[18] M. D. Peraro and F. G. van der Goot, "Pore-forming toxins: ancient, but never really out of fashion," Nature Reviews Microbiology, vol. 14, no. 2, pp. 77-92, 2016.

[19] M. Bischofberger, I. Iacovache, and F. Gisou van der Goot, "Pathogenic pore-forming proteins: function and host response," Cell Host \& Microbe, vol. 12, no. 3, pp. 266-275, 2012.

[20] V. Idone, C. Tam, and N. W. Andrews, "Two-way traffic on the road to plasma membrane repair," Trends in Cell Biology, vol. 18, no. 11, pp. 552-559, 2008.

[21] M. Husmann, E. Beckmann, K. Boller et al., "Elimination of a bacterial pore-forming toxin by sequential endocytosis and exocytosis," FEBS Letters, vol. 583, no. 2, pp. 337-344, 2009.

[22] D. G. J. Larsson, A. Andremont, J. Bengtsson-Palme et al., "Critical knowledge gaps and research needs related to the environmental dimensions of antibiotic resistance," Environment International, vol. 117, pp. 132-138, 2018.

[23] Y. Tu, "Artemisinin-a gift from traditional Chinese medicine to the world (nobel lecture)," Angewandte Chemie International Edition, vol. 55, no. 35, pp. 10210-10226, 2016. 